\title{
Perspective
}

PERSPECTIVE Actualité en histoire de l'art

4 | 2007

Genre et histoire de l'art

\section{Griselda Pollock : Féminisme et histoire de l'art}

Jacqueline Lichtenstein et Griselda Pollock

\section{(2) OpenEdition}

Journals

Édition électronique

URL : http://journals.openedition.org/perspective/3564

DOI : 10.4000/perspective.3564

ISSN : 2269-7721

Éditeur

Institut national d'histoire de l'art

Édition imprimée

Date de publication : 31 décembre 2007

Pagination : 568-584

ISSN : 1777-7852

\section{Référence électronique}

Jacqueline Lichtenstein et Griselda Pollock, « Griselda Pollock : Féminisme et histoire de l'art »,

Perspective [En ligne], 4 | 2007, mis en ligne le 31 mars 2018, consulté le 01 octobre 2020. URL : http:// journals.openedition.org/perspective/3564; DOI : https://doi.org/10.4000/perspective.3564

Ce document a été généré automatiquement le 1 octobre 2020. 


\title{
Griselda Pollock : Féminisme et histoire de l'art
}

\author{
Jacqueline Lichtenstein et Griselda Pollock
}

\section{NOTE DE L'ÉDITEUR}

Lors d'un débat public qui a eu lieu le 26 octobre 2007 à l'INHA, Griselda Pollock a répondu aux questions que Jacqueline Lichtenstein, professeur de philosophie à l'université de Paris-IV-Sorbonne, lui avait envoyées par écrit à sa demande, puis un débat s'est engagé.

1 Les études sur les questions de genre, qui connaissent depuis les années 1970 un grand succès dans les pays anglo-saxons, reçoivent un accueil plus réservé chez les historiens de l'art en France. Alors que les recherches de Linda Nochlin sont relativement bien diffusées en français (Femmes peintres 1550-1950 [1976], Paris, 1981; Femmes, art et pouvoir et autres essais, Nîmes, 1993), les travaux et réflexions de Griselda Pollock sont encore très peu publiés ( Histoire et politique: l'histoire de l'art peut-elle survivre au féminisme? ", dans Yves Michaud éd., Féminisme, art et histoire de l'art, Paris, 1994 ; « Où est l'interprétation? ", dans Régis Michel éd., Où en est l'interprétation de l'œuvre d'art?, Paris, 2000, p. 41-96 ; les premières pages de son ouvrage Differencing the Canon : Feminist Desire and the Writing of Art Histories, dans les Cahiers du genre, 43, 2007). Pourtant, par une approche critique qui articule psychanalyse, féminisme et études postcoloniales, elle a profondément renouvelé l'histoire de l'art.

2 Aussi la revue Perspective a-t-elle souhaité inviter Griselda Pollock pour l'entendre exposer son parcours et sa méthode, la façon dont elle se situe dans les études féministes et les questions de genre, et pour ouvrir le débat sur cette démarche qui a inauguré de nouveaux horizons. Elle a pu ainsi préciser ce qu'elle appelle les « interventions féministes en histoire de l'art». 
JACQUELINE LICHTENSTEIN. L'affluence montre que, contrairement à ce que je craignais, il n'est peut-être pas nécessaire de présenter Griselda Pollock en France. Contrairement à ce que je craignais parce que - ce qu'on appelle, pour aller très vite - les gender studies, puis les queer studies, etc., ne sont pas vraiment introduites en France, en tout cas pas sur le plan institutionnel ou universitaire, comme aux États-Unis ou en Angleterre. Les ouvrages de Griselda Pollock, que je suis ravie de rencontrer, ne sont pas non plus traduits en France, exception faite des trois articles mentionnés. Mais le fait que, dans cette immense liste de publications, d'articles, d'ouvrages de Griselda Pollock', presque rien n'ait encore été traduit, dit quelque chose sur le décalage en France, et peut-être qu'on essaiera d'en comprendre les raisons. Parmi toutes vos publications sur des thèmes assez variés, je voudrais revenir sur deux livres: Vision and Difference, publié en 1988, et Differencing the Canon, publié en 19992.

Mais pour introduire la séance, je souhaiterais d'abord vous poser une question assez générale comment définiriez-vous une histoire de l'art féministe?

Griselda Pollock. Pour entrer dans le débat, de manière un peu brusque peut-être... : il n'existe pas à mes yeux d'histoire de l'art féministe. Je ne peux pas la définir parce que je ne veux pas que mon projet soit enfermé, réduit à une tendance supplémentaire parmi une taxinomie des minorités dans le champ disciplinaire de l'histoire de l'art. Ce que je fais n'est pas une histoire féministe de l'art, ni une histoire de l'art féministe, ce sont plutôt des interventions féministes dans les histoires de l'art. J'insiste sur cette pluralité des histoires possibles, toujours à venir, sans qu'il y ait une seule vision face à laquelle toutes les autres interprétations paraissent transgressives, excessives, étranges, voire hostiles à une vérité unique. Je suis d'accord avec la pensée de l'artiste américaine Mary Kelly qui, en 1976, a refusé de se ranger dans la catégorie des artistes féministes ${ }^{3}$; en revanche, elle propose une «problématique féministe» qui ouvre des questions, qui demande des méthodes nouvelles, qui promet des découvertes, parce que la question de la différence sexuelle, de la subjectivité, de la sociabilité nous intéresse tous.

Il y a longtemps, j'ai avancé l'idée que l'histoire de l'art comme discipline ne pourrait pas survivre au défi lancé par les questions féministes ${ }^{4}$. On doit d'abord reconnaître la critique lancée par le féminisme, qui identifie un inconscient idéologique - d'après la proposition de Frederic Jameson selon laquelle il existe un inconscient politique à la base de la littérature ${ }^{5}$ - et étaye le sens de l'histoire de l'art actuelle. Suivant la lecture que fait Sarah Kofman de la pensée esthétique de Freud dans son livre L'enfance de l'art (Paris, 1970), j'ai proposé dans Differencing the Canon (1999) d'expliquer, examiner, analyser la ténacité du canon masculin. Pourquoi existe-t-il toujours, même après les critiques des féministes ? Et j'ai répondu que, comme Sarah Kofman nous l'enseigne, il y a une structure psychique, à la fois narcissique et théologique, dans l'idéalisation des artistes, qui est au cœur de l'histoire de l'art moderne. C'est une idéalisation perpétuelle du masculin dans la figure du héros ou du père, figures auxquelles l'artiste est identifié, au lieu d'une attention désintéressée et impartiale pour toutes les productions culturelles. Donc, un désir homosocial, et une identification et une idéalisation ambivalentes maintiennent un canon exclusivement masculin, présenté néanmoins comme un fait naturel.

Les questions féministes ont donc commencé, pour moi, par une interrogation du discours de l'histoire de l'art. Bien avant que je ne rencontre les écrits de Foucault ou de Derrida, j'ai élaboré une déconstruction du langage de l'histoire de l'art et une analyse des formations discursives dans lesquelles on peut lire le refoulement, la suppression et l'exclusion qui découlent de l'opposition binaire entre la masculinité, terme toujours conçu comme positif, et le féminin. Le féminin, la féminité, telle 
qu'elle est inventée par l'histoire de l'art, ne peut jouer un rôle que comme miroir négatif, un signe d'infériorité, de manque. Or les termes "masculin » et "féminin » n'ont aucune signification en eux-mêmes, ils ne sont que des oppositions qui font sens dans une hiérarchie. Le second terme est toujours vide, insignifiant - mais le signifié n'est pas une femme, une personne. Il suffit à «la femme » de jouer ce rôle réflexif pour que le soi, le moi, le masculin puisse être solidifié face à cet autre. Pour le titre de mon premier livre, old Mistresses: Women, Art and Ideology (avec Rozsika Parker, Londres, 1995), je me suis inspirée de deux historiennes d'art américaines, Ann Gabhart and Elizabeth Broun, qui l'avaient choisi pour la première exposition sur les artistes femmes depuis le Moyen Âge jusqu'au XvIII ${ }^{e}$ siècle, intitulée Old Mistresses: Women Artists of the Past (Baltimore, Walters Art Gallery, 1972). Et que voulaient-elles dire? L'expression «old mistresses " n'évoque pas la notion très honorable de «old masters ", mais plutôt l'idée d'amantes âgées et rejetées. S'il n'existe pas d'expression dans notre langue pour signifier le même respect ou la même déférence pour les artistes/femmes que celui dont jouissent les « vieux maîtres » et si, pour indiquer que l'artiste est aussi une femme, nous devons ajouter le qualificatif «femme » ou l'adjectif «féminin », nous devons admettre que la position de l'artiste et de l'art n'est jamais neutre. Elle est toujours déjà entièrement occupée par le masculin qui se pose comme étant universel, et donc neutre. L'histoire de l'art participe donc de la production du genre, même si elle se présente comme objective. Ainsi que Teresa de Lauretis nous l'enseigne ${ }^{6}$, l'histoire de l'art, comme toutes les choses de la culture, relève déjà du genre. Elle est profondément partie prenante de la production et la répétition par lesquelles la hiérarchie et la dissymétrie des pouvoirs, des valeurs et des identités que nous comprenons comme genre sont produites et sans cesse renouvelées. Étudier l'histoire de l'art de manière traditionnelle, c'est se soumettre à une méthodologie phallocentrique, que l'on soit homme ou femme.

Mon ambition n'est donc pas l'intégration d'une minorité féministe qui s'occupe des femmes - les artistes "inférieurs" - ou des questions ponctuelles de genre qui intéressent seulement les femmes. C'est d'abord de comprendre l'histoire du féminisme comme un fait de l'époque actuelle, le produit et l'effet des crises de la modernité, qui sont visibles aussi dans les luttes continuelles contre le racisme, l'homophobie, l'antisémitisme, bref les luttes pour la réalisation profonde de ce qui a été promis mais non livré avec la Déclaration des droits de l'homme, assez ancienne maintenant. Au moment, à la fin du XVIII ${ }^{e}$ siècle, où l'on commençait à penser à l'être humain, l'humanité, cette promesse a été nommée les "droits de l'homme», et donc on a recréé, sur le plan de la modernité, une inégalité des sexes: on a refondé le genre de nouveau.

Deuxièmement, on a une histoire de l'art que l'on sait commencer au XVIII siècle avec une contradiction entre deux termes. D'un côté les philosophes ont identifié l'art comme catégorie singulière, universelle, absolue, et l'art est donc devenu, pour la philosophie, une question de jugement et d'expérience au-delà de la pensée (aisthèsis) . En même temps, on a proposé que cet absolu, cet art qu'on peut juger avec une norme universelle, puisse avoir une histoire, être historiquement variable. Comment réconcilier cette contradiction entre l'absolu et le contingent ? Pour Winckelmann, la réconciliation passe par l'idéalisation de l'art grec comme norme, comme canon suprême, même s'il a proposé d'y voir le résultat de la politique, du climat, de la géographie de la Grèce antique. 
Dans un deuxième temps, les historiens de l'art ont tenté d'écrire une histoire universelle, mais elle s'est constituée d'un point de vue historique et politique, c'està-dire du point de vue des écrivains et philosophes allemands et européens, et donc marqués par leur politique, leur climat ou plutôt leur géographie mentale. Or la géographie mentale du XIX ${ }^{\mathrm{e}}$ siècle, c'est celle de l'État-nation. Cette histoire de l'art reflète donc les aspirations nationalistes des peuples de l'Europe. De Franz Kugler à Charles Blanc ${ }^{7}$, on a produit une histoire des écoles nationales, soutenue par une philosophie hégélienne qui était progressive et téléologique. Ces grandes histoires des écoles allemande, française, anglaise et espagnole sont à la fois racistes, antisémites et antiféministes.

Une troisième génération d'historiens de l'art peut être identifiée dans la dernière décennie $d u x x^{e}$ siècle et au commencement $d u x^{e}$ siècle. C'était un moment de grande spéculation théorique et d'invention de méthodes et de concepts par des esprits d'une grande profondeur intellectuelle, de Wölfflin à Riegl, Dvořák, Antal, Warburg. À cette époque, l'histoire de l'art était contestée de l'intérieur par des écoles diverses, à Vienne, Hambourg, Paris, Berlin, Munich, en Suisse ou à Budapest, mais non à Londres. La grande question était d'élaborer la spécificité de l'art identifiée comme la forme, visible à travers le temps, dans une temporalité, en fonction des changements de styles, avec Wölfflin, par exemple. Mais cette nécessité de sauver la particularité de l'art plastique d'une immersion burckhardtienne dans la culture - ou dans l'histoire en général - a été remise en cause par l'interrogation symbolique, et même psychologique de l'art entreprise par Aby Warburg, qui propose un système vraiment complexe - l'iconologie. Ces générations de grands penseurs participent à un moment que je nomme les révolutions de 1905. C'est le moment d'Einstein, Saussure, Husserl et Freud. C'est aussi un moment de féminisme culturel militant, qui marque l'arrivée des femmes sur le devant de la scène du modernisme. Ces penseurs ont modernisé les études de la physique, du langage, de la philosophie et mis en avant la subjectivité humaine. Le choc de leurs pensées révolutionnaires n'a pas été absorbé par notre culture avant le milieu du $\mathrm{xx}^{\mathrm{e}}$ siècle, avec ce que nous identifions maintenant comme le moment structuraliste puis poststructuraliste. L'histoire de l'art s'est placée dans une position ambivalente à ce moment-là. D'un côté, elle était absolument transformée, sinon traumatisée, par l'arrivée du fascisme, par l'expulsion et l'émigration forcée de tant d'historiens de l'art dans les grands centres européens et par les effets que cela a produits sur eux, parce qu'ils avaient été persécutés pour leurs identités ethniques. L'histoire de l'art fondée par Panofsky et les autres historiens qui ont émigré aux États-Unis a résisté à toutes les questions sociales pour embrasser une idée rédemptrice de l'art et pour considérer l'histoire de l'art comme un humanisme éducateur et civilisant. Après la guerre, les contestations et les débats entre formalistes, marxistes et warburgiens ont été déterminés aussi par un autre traumatisme, celui de la guerre froide. Au milieu du xxe siècle, l'histoire de l'art, notamment anglophone, s'était restreinte au formalisme, sans aucune référence à l'histoire sociale et politique ou aux autres idées intellectuelles de ce moment-là. Au début des années 1970, les débats ont repris parce que des étudiants en histoire de l'art comme moi ont regardé en arrière pour se demander pourquoi les moyens offerts pour étudier quelque chose d'aussi complexe que l'art et ses histoires étaient devenus si maigres, si anti-intellectuels, si conformistes et si repliés sur la seule discipline. Pour ma part, je m'interrogeais sur la contradiction entre ce que je voyais 
dans les œuvres, en tant qu'historienne et artiste moi-même, et les moyens que m'offraient les professeurs pour l'étudier et le comprendre. Ils se résumaient à peu de choses près à ce schéma de Barr où la période 1910-1940 est vue seulement comme une chronologie, sans la guerre mondiale ni l'arrivée du fascisme, avec seulement une histoire de développement stylistique.

Mais dans les années 1970, de nouveaux mouvements sociaux éclatent, en particulier le mouvement des minorités, qui brisent les barrières entre les états nationaux, demandent une autre fondation des connaissances, imposent une nouvelle forme de politique et font voler en éclats les compartiments de la vie, de la connaissance, de l'art, de l'histoire, du public, du privé. Avec cela déferle une nouvelle vague de féminisme qui formule une repolitisation de la question du genre refoulée par le modernisme institutionnalisé. On doit donc reconnaître là un événement historique d'une grande importance, un phénomène de révolte et de révolution qui n'était pas confiné à l'espace de la rue ou aux médias uniquement politiques. Et, plus important encore, pour la première fois le genre trouvait une articulation théorique ${ }^{8}$. Parallèlement, le mouvement féministe a suscité pour la première fois dans l'histoire une densité significative de femmes à l'université, comme professeures et comme étudiantes, qui pouvaient parler de leur propre voix sur les questions de leurs propres histoires. Le féminisme a créé une conscience d'être quelque chose de particulier et pas seulement cet autre, mutilé, déficient, qui ne doit aspirer qu'à nier la féminité et à assimiler la masculinité universalisante, pour que nous, femmes, puissions être reconnues comme les égales des hommes: le féminisme a pu développer une pensée qui prenne en compte la différence, entre représentation et sexualité, et ce sur un plan philosophique. Cela nous avait déjà été proposé par Simone de Beauvoir en $1949^{\circ}$, mais c'est seulement avec l'arrivée du féminisme comme mouvement intellectuel et théorique qu'on a pu utiliser ses idées.

J. L. Comment cette histoire de l'art féministe se distingue-t-elle des gender studies ou des queer studies?

G. P. Sur la différence entre les gender studies et la " problématique féministe ", sur la question de la différence sexuelle, il n'existe aucune unanimité entre les féministes. Selon Joan $\operatorname{Scott}^{10}$, par « genre », on doit comprendre « axe de pouvoir ». Mais, pour le sens commun, le mot ne semble être qu'une description spontanée des deux types de genre, deux sexes, déterminés par leur fonction et par leur anatomie reproductive. En revanche, pour les sociologues, Bourdieu notamment ${ }^{11}$, gender peut vouloir désigner les constructions sociales imposées à ces anatomies pour socialiser nos corps et nos esprits selon les attentes de la société. On a donc des analyses de genre social pour signifier les rôles que telle ou telle société impose à ses populations. À l'opposé, dans les discours vraiment féministes, nous proposons l'idée que le genre n'existe pas en soi. C'est précisément le résultat de procédés sociaux, linguistiques, culturels qui produisent, uniquement par les répétitions que nous-mêmes effectuons, des effets qui sont hétérosexualisants et, si je peux faire ce jeu de mots, « engendrants ». C'est vraiment quelque chose d'artificiel. Dans quel but ? Pour que la société puisse se reproduire, pour produire l'effet de la féminité et de la masculinité dans une hiérarchie asymétrique nécessitée par les structures de parenté pour effectuer l'échange de biens sur lequel la culture repose. Avec pour résultat de 
nombreuses formes de sexualités sont totalement mises à l'écart pour que cette représentation des êtres humains selon ces deux genres puisse prendre toute la place. La création du champ académique des gender studies semblerait promettre une égalité des études portant sur la masculinité et la féminité, ce qui paraît beaucoup plus généreux que le féminisme qui, apparemment, ne s'occupe que des femmes. Mais ce n'est pas vrai. Pour moi, le danger, c'est que les gender studies répètent sans critique ce que la pensée féministe met en cause, c'est-à-dire la présentation des deux genres comme deux entités égales. Or on doit se demander ce que cette répétition refoule en insistant sur le naturalisme des deux genres : elle limite la diversité des sexualités, la diversité des possibilités humaines non réglées par la famille et la hiérarchie des sens et des sexes nécessitée par la seule reproduction. Or si on veut se demander « qu'estce que la femme? " et "qu'est-ce que l'homme?», on doit résister à cette idée de genre parce que cela désigne toujours seulement «l'homme » et « la femme » à la place des possibilités beaucoup plus complexes, beaucoup plus diverses, beaucoup plus temporelles et susceptibles de modifications.

Quant aux queer studies, profondément influencées par la pensée de Judith Butler ${ }^{12}$, elles voudraient déconstruire cette supposition hétéronormative sur laquelle reposent les gender studies, même si ces dernières accueillent volontiers ceux qui veulent changer de genre - transgender - ou effectuer toute autre possibilité de transformation. Mais ce que nous perdons dans les queer studies, c'est la spécificité de l'«autre » féminin, qui est toujours refoulé par un phallocentrisme incontesté, même dans les queer studies, du moins quand les queer studies n'évoluent pas au contact du féminisme.

Finalement, qu'est-ce que la problématique féministe par rapport à celle des gender ou des queer studies? Et pourquoi cette problématique entre-t-elle dans le cadre des études de l'art et de ses histoires? Si la pensée féministe est de nature transdisciplinaire - car elle ne peut être que transdisciplinaire - il y a aussi une spécificité de la pensée féministe esthétique et culturelle. Nous sommes formés par des représentations, mais nous agissons, nous changeons ces normes par nos créations poétiques et artistiques. Et donc l'art se place toujours en même temps sur le plan idéologique et aussi sur le plan transgressif. Savons-nous tout ce qui nous manque si nous refusons d'écouter la voix des femmes, de conserver leurs archives, d'étudier leurs pensées, si nos bibliothèques et nos musées sont à moitié vides ? C'est une catastrophe pour tous. Un héritage amputé, une culture monosexuelle sont aussi infertiles et stériles qu'une culture monoethnique ou monosociale. Et si nous pensons que nous avons déjà un riche héritage au Louvre, imaginez-vous la richesse que nous aurions eue avec les voix de l'humanité tout entière! Et le résultat de ce refoulement actif dans les archives, de cette amnésie créée, de cet oubli volontaire de ce qu'ont fait les femmes, les omissions actives par les historiens, les conservateurs, c'est que nous ne savons pas encore ce que peut être la différence des femmes. Il ne s'agit pas simplement de réparer l'absence avec les noms des femmes qui ont écrit ou peint dans le passé. Les formations intellectuelles de notre discipline sont déjà phallocrates, comme le dit la poétesse américaine Adrienne Rich, dans son essai "Towards a Woman-Centered University" $(1978)^{13}$ où elle constate à quel point l'université est centrée sur les hommes: nos modes d'évaluation eux-mêmes, nos moyens de recherche ne sont pas neutres. On doit donc se représenter la féministe comme une archéologue qui voyage dans l'art du passé mais sans les clés pour 
déchiffrer les inscriptions du féminin qu'elle rencontre sur des objets, des textes, des monuments. Nous devons apprendre à déchiffrer les traces du féminin déjà inscrites sur les textes de la culture par les femmes, par les femmes-hommes, par les nonhommes ${ }^{14}$, qui n'ont pas eu à leur disposition les moyens complets pour comprendre ce qu'elles ont fait. La féminité ne doit pas être comprise comme essence ou comme nature, mais comme un effet variable qui change très lentement et sur une très longue durée, un effet des éléments psychiques, linguistiques, culturels vécus en esprit et dans le corps. Sans les outils culturels et les mots nécessaires pour la comprendre, la féminité reste inconnue par les femmes elles-mêmes, même si elles l'inscrivent, inconsciemment, dans leurs "sextes $»^{15}$. Les produits culturels sont une forme de poièsis, de création, et offrent toujours une trace en excès du sens immédiat. Donc comme un analyste écoutant le mouvement de l'inconscient, l'historien de l'art féministe cherche les traces des inscriptions du féminin inconnu. Mais pour l'interprétation, de même que chez Freud, nous avons dû créer un vocabulaire, des concepts nouveaux et travailler lentement, par petites étapes, pour construire plusieurs ressources théoriques et pratiques dans les disciplines apparentées. Nous avons dû construire des modes d'analyse performants et sensibles au discours de l'autre: le féminin comme autre mais aussi le familier refoulé par la phallocratie. Dans ce passage réflexif, nous devons prêter aux textes et aux images des femmes nos propres histoires, même si, nous-mêmes, nous ne les comprenons pas. Nous devons prêter nos expériences et nos mémoires à l'art de l'autre, même si celui-ci est déformé par l'absence des formes de reconnaissance que pourrait élaborer une culture tissée selon les désirs et les fantasmes des femmes. J'ai donc proposé dans le livre Generations and Geographies in the Visual Arts ${ }^{16}$ un moyen de penser les artistes femmes sans les soumettre à une catégorie homogène : on doit les représenter pour affirmer que chacune a une voix singulière. Et $\mathrm{j}^{\prime}$ insiste, comme Julia Kristeva ${ }^{17}$, sur le fait qu'il ne s'agit pas de trouver l'aspect collectif de la féminité, mais d'identifier la singularité de chaque femme dans chaque génération, dans chaque position géographique, sociale ou historique. Comme le dit Shoshana Felman, une critique de la littérature d'orientation lacanienne :

"Je suis d'avis qu'aucune d'entre nous, en tant que femme, ne possède pour l'instant de vraie autobiographie. Habituées à nous traiter nous-mêmes en objets, à être positionnées comme autres, et à nous considérer comme étrangères à nousmêmes, nous disposons d'une histoire qui par définition ne peut être présente à nous-mêmes, une histoire qui, en d'autres termes, n'est pas une histoire, mais doit en devenir une. Et cela ne peut avoir lieu qu'en scellant un pacte de lecture, c'est-àdire en passant par l'histoire de l'autre, l'histoire lue par d'autres femmes, l'histoire d'autres femmes, dans la mesure où cette histoire de l'autre, de même que notre propre autobiographie, nous devons encore nous l'approprier. Cette appropriation ne peut pas se faire, je pense, en prenant un cheminement direct vers nous-mêmes en tant que femmes - en nous abordant sur un mode personnel -, ou en laissant de côté la culture et en nous éloignant en dehors du texte. Je propose plutôt que nous puissions créer et accéder à notre histoire uniquement de façon indirecte, en conjuguant la littérature, la théorie et notre propre autobiographie par l'acte même de la lecture, et en sollicitant le 'texte' qu'est la culture pour nous rendre compte de l'absence à la fois de notre différence sexuelle et de nos propres autobiographies $»^{18}$.

Donc, je ne peux pas définir l'histoire de l'art féministe, parce qu'elle est à venir. Ce que nous avons fait jusqu'à maintenant, ce ne sont que des préliminaires pour identifier l'énormité de notre travail qui consiste à relire toute l'histoire de l'art - car 
nous sommes conscientes que les questions de la production de genre et les jeux constants de la différence sexuelle peuvent nous faire déboucher sur des choses inconnues et inouïes.

\section{J. L. Quels sont les enjeux politiques d'une histoire de l'art féministe?}

G.P. Le féminisme est une critique du phallocentrisme, alliée à la critique du colonialisme, du racisme, de l'homophobie, du fascisme et de l'antisémitisme. Il est la critique d'un système historique, même si celui-ci est un état de longue durée. Comme le dit J. Kristeva, le temps de la reproduction et de la sexualité, qu'elle nomme «le temps des femmes ${ }^{19}$, est d'une temporalité différente de celle de l'histoire des nations et de la politique. Pour moi, le féminisme participe à une lutte historique qui a commencé il y a un siècle pour la modernisation de la différence sexuelle. Cet événement se déroule à travers l'étude de l'art parce que l'art est à la fois une activité symbolique et esthétique, affective, éducatrice des émotions, et l'un des moyens par lesquels nous nous reconnaissons : un miroir des représentations de nous-mêmes. Le féminisme nous permet d'embrasser l'ensemble de notre espèce, mais une espèce caractérisée, comme le dit Hannah $\operatorname{Arendt}^{20}$, par la pluralité, la diversité et les difficultés de la condition humaine. Le projet du féminisme dans la culture, comme dans la pensée critique et poétique, est de résister à l'enfermement, par inconscience ou idéologie, dans un couloir étroit et froid, monosexuel, hétéronormatif, ethnocentrique, christianocentrique, raciste. C'est une volonté et une tentative de transformer la culture entière, de risquer la mort pour la vie, de déplacer le thanatos pour cultiver l'éros. Nous ne sommes pas une voix mineure mais une force qui exige la vastitude de la science et qui promet de nous libérer des effets mortifiants d'une phallocratie incontestée. Les enjeux politiques, pour nous, dans l'art et pour la féminité, sont à mon avis vraiment une question de vie et de mort du passé et de l'avenir.

J. L. Ne croyez-vous pas que cette approche laisse échapper ce qui fait la spécificité des productions artistiques au sein des autres productions culturelles?

G.P. Selon la théorie élaborée par les psychanalystes féministes, l'esthétique peut être située au même niveau psychique archaïque que l'origine de la sexualité humaine ou de la différence sexuelle. Le travail féministe sur la question de l'esthétique s'occupe des effets d'une rencontre phénoménologique et spéculaire (dans le sens de la vision) entre une personne en chair et en os et l'œuvre matérialisée en art. Je propose donc que l'œuvre d'art ne soit pas un objet de valeur en soi, mais un événement intersubjectif, sinon transsubjectif. Le féminisme n'est pas du tout hostile aux questions esthétiques, même s'il y a des tendances dans la visual culture qui s'adressent seulement aux questions de l'image et des régimes de représentation, et non à la dimension plastique. En ce moment par exemple, précisément parce que l'art contemporain lui-même a abandonné les pratiques traditionnelles - la peinture et le dessin, pour ne citer qu'eux - pour s'occuper de l'image, de la cinématique, de l'objet réel, de l'installation, de la réalité, de la virtualité, de la culture médiatique et cybernétique, les oeuvres des artistes actuels 
qui produisent des peintures et des dessins comme Bracha Ettinger, Christine Taylor Patten, Judith Tucker, Ida Appelbroog, etc. m'intéressent beaucoup car les significations de ces matériaux ont vraiment changé dans leur rapport à notre culture assez médiatique. Et les gestes de la peinture ou du dessin, qui ont une temporalité et un lien direct avec le corps qui travaille, sont devenus bien différents de ceux qui avaient cours au moment où la peinture était le moyen d'expression par excellence, de la moitié $d u x^{e}$ siècle jusqu'au milieu du $x^{e}$ siècle. Avec le postmodernisme, l'ère de la peinture est finie, mais elle survit comme quelque chose d'anachronique et donc de très intéressant. Nous lisons ces marques de l'art pour autre chose que pour les traces de l'artiste que l'on peut idéaliser ou pour les styles que l'on peut classer en périodes et mettre en rapport les uns avec les autres. On les lit pour ce qu'elles montrent des mouvements créateurs, poétiques, d'un corps, d'une subjectivité, d'un inconscient sémiotique. La différence avec la visual culture se trouve donc dans les moyens d'interprétation - même si nous partageons le même territoire des images -, et dans l'intérêt pour l'aspect psycho-esthétique de l'art.

J. L. Merci beaucoup pour ces réponses qui montrent la richesse et la complexité de votre démarche, mais continuent de m'intriguer. Par exemple, vous avez fait un jeu de mots sur gender et engendrer qui est tout à fait légitime en anglais mais ne correspond à rien, ne produit aucun effet de sens en français. J'ai trop travaillé sur la question de l'intraductibilité, c'est-à-dire sur ce qui se passe quand on ne peut pas traduire un concept d'une langue à une autre, et sur la manière dont toute traduction inscrit le concept dans une nouvelle configuration pour ne pas prendre au sérieux le problème que soulève ici la différence des langues. Pourquoi le mot "genre» ne fonctionne-t-il pas en français? La revue Les cahiers du genre, j'ai compris ce que ça voulait dire non quand j'ai entendu pour la première fois le titre, mais seulement quand je l'ai pensé en anglais. II y a des spécificités de langues qui sont des spécificités de pensées, des spécificités de catégories mentales, d'appréhension du monde, etc. "Engendrer " renvoie à "générique »... et non à "genre » au sens français du terme. Ce n'est pas du tout un argument contre les gender studies, mais je crois que cela peut aider à comprendre des différences.

J'ai beaucoup aimé votre précision tout au début quand vous dites qu'il n'y a pas d'histoire de l'art féministe mais des interventions féministes en histoire de l'art, ce que vous dites d'ailleurs dans vos livres à plusieurs reprises: au fond il ne s'agit pas pour vous d'ajouter une histoire de l'art féministe à toutes les formes d'histoire de l'art qui existent déjà, mais de déconstruire - vous employez le mot - l'histoire - ou les histoires - de l'art par une intervention ou un point de vue féministe. Là, vous employez le mot «intervention ». Je saisis tout à fait l'intérêt et la pertinence de ces interventions, mais je ne suis pas sûre de toujours saisir la fonction déconstructrice, sur des cas précis, d'une approche féministe.

J'ai également été très frappée par votre approche disons très hégélienne de l'histoire de l'art. Même si je tiens compte du fait qu'une présentation orale comme celle que vous venez de faire vous contraint inévitablement à schématiser votre pensée, il n'en reste pas moins que l'immense tableau de l'histoire de l'art à partir de Winckelmann que vous nous avez dressé témoigne d'une volonté de trouver un sens et une cohérence, une rationalité, dans le réel historique, typique de toutes les approches disons idéologiques, politiques ou féministes de l'art, qui visent toutes, peu ou prou, à construire un grand récit cohérent. Ce qui me gêne dans votre vision de l'histoire de l'art, c'est précisément qu'elle correspond à une construction narrative, un peu trop cohérente à mon goût. Comme me gêne, dans toutes les approches sociologiques, politiques, idéologiques, etc., et donc dans la vôtre, le fait de traiter l'art exclusivement comme une production culturelle, sans prendre en compte ce que j'appellerai la spécificité de l'art au sein des productions culturelles. Que l'art soit une production culturelle, c'est évident. Et donc que l'art, en tant qu' artefact, produit de l'activité humaine, ait un sens, comme vous le dites, c'est évident. Toutes les productions culturelles ont un sens. Mais ça, c'est hégélien! Je ne veux évidemment pas dire que Hegel, ce n'est que ça. C'est le point de vue sur l'art de la Kulturgeschichte, et on le retrouve dans toutes les approches sociologiques ou historiques de l'art, qui traitent l'art essentiellement comme document permettant de comprendre, d'éclairer une époque, des mentalités etc. Toutes 
ces approches ont d'ailleurs en commun d'adopter une position fondamentalement relativiste... Alors, peut-être que ce n'est pas la tâche de l'histoire de l'art, ou plutôt de l'historien de l'art de prendre en compte et de rendre compte de la spécificité de l'art. Mais c'est pourtant cette spécificité, ce qui fait qu'un tableau n'est pas un "produit culturel » comme un autre, ou encore, pour le dire autrement, que ce que l'on entend et désigne par le terme "art " ne recouvre pas exactement ce que l'on entend par le terme "culture", qu'une œuvre artistique ce n'est pas un simple produit culturel, contrairement à ce que disent nos ministres et plus généralement les politiques.

G. P. Pour moi, dans l'histoire, il y a toujours cette tension, cette opposition, cette contradiction entre le moment où l'on peut penser l'art comme catégorie philosophique ou comme production spécifique... C'est pour cette raison que je proposais l'idée que les trois générations d'historiens que j'ai présentées très rapidement sont des moments différents de ces contradictions.

Je voudrais insister sur le fait que l'intervention féministe n'agit pas seulement sur le symbolique comme quelque chose de culturel. C'est pourquoi je me suis orientée, finalement, vers la question de l'esthétique : que se passe-t-il dans cette pratique qui n'arrive nulle part ailleurs ? Qu'est-ce qui est possible et qu'est-ce qui est arrivé avec la psychanalyse, les événements de l'histoire de l'art, les mutations en art contemporain, qui nous indique qu'on peut définir l'art comme processus spécifique mais pas seulement dans le sens philosophique ? Kant et Baumgarten ont commencé à définir l'esthétique mais enfin, selon moi, il y a un problème esthétique "après Auschwitz ", des esthétiques modifiées par les questions que pose la différence sexuelle. Je ne voudrais pas qu'on voie l'histoire de l'art féministe - l'intervention féministe - seulement comme une approche imposée par les visual studies, c'est-à-dire l'étude des représentations, de l'image, des discours idéologiques de cette visual culture. Mais en histoire de l'art, nous sommes toujours chargés de repenser ces problèmes, au sein de quelque chose qui change, qui est vraiment historique et donc doit avoir à faire avec la société et les changements économiques et politiques, mais qui n'est pas identique à ça.

J. L. Vous avez raison, l'art est une catégorie philosophique, abstraite, mais qui a quand même ceci de très particulier que c'est une catégorie qui fonctionne toujours, dès le début (il n'y a pas si longtemps que ça, disons depuis la fin de la Renaissance, quand on est entré dans l'identification des arts avec les arts libéraux), à la fois et indistinctement, comme une catégorie descriptive et comme une catégorie évaluative. Quand on dit d'un objet que c'est de l'art, cela veut dire que c'est descriptif, que c'est de l'art parce que c'est dans les musées etc. Mais en même temps, c'est une catégorie évaluative. Alors on peut en discuter, il y a des philosophes aujourd'hui qui discutent, qui contestent le caractère évaluatif du concept d'art et qui disent : "après tout, 'art', ça ne veut pas dire que c'est bien. Le mauvais art, c'est de l'art aussi ». Pourquoi est-ce que je vous parle de ça? Parce que, par exemple, à un moment - je crois que c'est dans Vision and Difference -, vous dites qu'au fond il y a trois mensonges - ou trois erreurs - contre lesquels il faut lutter ou qu'il faut réfuter. Le premier, c'est qu'il n'y a pas eu de femmes artistes. Donc on pourrait refaire une histoire qui redonne la place à cela. Mais ce qui m'intéresse, c'est votre deuxième proposition: que les femmes artistes sont de second niveau, "second rate ». C'est aussi une proposition qu'il faut réfuter. C'est-à-dire, quand il y a eu des femmes artistes, elles étaient des femmes de niveau inférieur. À ce moment-là, comment pouvez-vous éviter la question des critères d'évaluation et la question du jugement de valeur de l'art? Vous l'évitez comme tous les sociologues de l'art l'évitent, comme les historiens de l'art l'évitent systématiquement. On peut montrer qu'une œuvre d'art en tant que production culturelle a un 
sens, que c'est important, que ça exprime telle idée, que ça joue un rôle, etc. Mais on ne pourra pas éviter que l'art soit l'objet d'un jugement de valeur. Et donc, comment allez-vous démontrer que les femmes ne sont pas de second niveau? Je suis extrêmement frappée que les artistes -

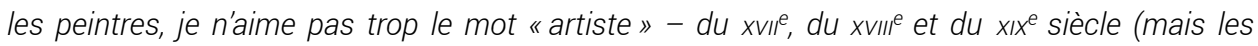
écrivains, c'est pareil) ont des critères extrêmement précis pour se juger, pour dire que c'est bon, que c'est mauvais, etc. Ils sont comme tout le monde, ils appartiennent à leur époque, ils sont racistes, sexistes, tout ce que vous voulez, comme les hommes de leur temps, bien évidemment ! Mais ils ont des problèmes plastiques à résoudre et se demandent comment ils peuvent le faire ; ils critiquent tel artiste parce qu'il a bien résolu ou mal résolu ce problème, etc. Et donc je ne pense pas qu'on puisse éliminer cette question des critères d'évaluation ou qu'on puisse la dissoudre entièrement dans une approche de type politique, idéologique, sexuelle ou féministe.

G. P. C'est une question que l'on trouve partout en ce moment. Au Danemark a lieu un grand débat sur ces problèmes de qualité et de discrimination parce le gouvernement a tenté, en 2003, de préciser l'héritage artistique danois ${ }^{21}$ afin de protéger la nation du processus transformatif des cultures importées et donc de la différence. Il y a également un débat intense en Suède. Le directeur d'un grand musée de ce pays a demandé au gouvernement une forte somme d'argent pour acheter des œuvres de femmes parce que depuis des décennies, voire un demi-siècle, on n'a pas acheté de créations de femmes artistes, parce que l'on achète seulement des choses qui ont une valeur indiscutable. Dans Differencing the Canon, j'ai exposé les tendances psychiques (narcissique et idéalisante) des hommes qui prennent comme valeur éternelle ce qui n'est, en vérité, que l'expression de leurs désirs.

Je ne refuse jamais le formalisme en soi - parce que c'est absolument nécessaire comme moyen d'approche -, mais le formalisme seul n'est pas suffisant pour reconnaître la complexité de l'art. Il ne s'agit pas de dire que Mary Cassatt est aussi intéressante que Degas, mais de créer la possibilité de voir pourquoi elle n'intéresse pas les hommes et comment je peux recréer un terrain commun entre les deux artistes. Heather Dawkins, par exemple, qui écrit sur Degas ${ }^{22}$, a introduit dans son analyse de la figure de la baigneuse la question du chagrin, de la douleur du peintre, car Degas a perdu sa mère à l'âge de 12 ans. Je m'intéresse beaucoup à l'effet psychologique de cette perte si précoce, dont on ressent l'effet dans l'ambiguïté de ses images de femmes. Si on arrive à voir Degas comme un artiste traversé depuis longtemps par le chagrin ou la douleur, on arrive à construire une vision différente de Degas et à le faire converser avec des artistes qui, comme Mary Cassatt, apparaissent sans intérêt pour l'histoire de l'art masculine. En outre, je peux replacer Mary Cassatt dans le contexte d'une des tendances culturelles de la fin du XIX ${ }^{\mathrm{e}}$ siècle, caractérisée par un intérêt pour l'intériorité psychologique et qui participait aux nouvelles recherches sur ce qu'est l'enfant par rapport à l'adulte, ce qu'est cet autre, cette espèce étrange qu'est l'enfant... On peut voir dans les œuvres de Cassatt non la maternité et la domesticité, mais les interrogations d'une fille-adulte-artistechercheur sur une expérience qu'elle n'a pas eue, la maternité, de même que Degas s'interroge sur quelque chose qu'il n'a pas vécu, comme les expériences de danseurs. On change l'angle de vue et l'interprétation pour faire entrer en jeu des aspects beaucoup plus intéressants, la complexité des êtres humains, au lieu de se dire qu'on regarde seulement les inventions plastiques ou les pastels de Degas. Et ce n'est pas une question de significations idéologiques ou de valeurs formalistes, mais ça se produit exactement et ne peut se produire que sur le plan plastique, et c'est ce que j'appelle la poièsis. C'est le moment de création absolue. Mais on doit emprunter à la psychanalyse, comme à la philosophie, l'institutionnalisme, la biologie, pour produire 
une histoire de l'art, une interprétation des possibilités de ce moment qui soit vraiment respectueuse de l'invention qu'était l'art. J'ai toujours en tête de tracer le mouvement de l'intelligence artistique articulée à des problèmes formels. C'est pourquoi le modernisme est une chose très intéressante, très complexe. À la fin, la penseuse féministe ne résiste pas aux questions de valeur dans l'art, mais modifie les moyens par lesquels on peut apprécier l'intelligence, l'invention et les effets symboliques aussi bien qu'esthétiques, de toutes les œuvres d'art - au lieu d'avoir seulement une image de Degas diminuée parce qu'on refoule une partie de la conversation, le dialogue qu'il a entretenu avec Cassatt ou d'autres.

J. L. Vous avez commencé en disant que vous êtes une historienne de l'art féministe, mais qu'il n'y a pas d'histoire de l'art féministe. Est-ce que vous diriez la même chose à propos de l'art, cette fois-ci, et pas de l'histoire de l'art? Vous citez dans un de vos textes l'artiste américain Katie Watt: fait-elle de l'art féministe? Est-ce que vous diriez la même chose pour l'art que pour I'histoire de l'art, c'est-à-dire : il y a des artistes féministes aujourd'hui mais pas d'art féministe, ou est-ce que vous pensez qu'il y a un art féministe, même s'il n'y a pas d'histoire de l'art féministe?

G. P. L'histoire de l'art est formée par les musées, qui ont toujours eu la nécessité de créer des catégories : mediums, périodes, écoles, mouvements, auteurs. Elle cherche donc toujours à placer l'art dans des catégories muséales. Dire " art féministe », c'est trouver un tiroir dans lequel enfermer l'art. Je ne suis pas une historienne des femmes mais une historienne de l'art, qui pose la question de la différence sexuelle mais aussi beaucoup d'autres - sur le postcolonialisme, l'homophobie, les sexualités. $\mathrm{Au}$ lieu de catégories comme l'« art féministe ", on doit penser à une problématique féministe. Je voudrais introduire le cas de Mary Kelly, que je situe comme une artiste contemporaine, et non américaine ou anglaise. Elle maintient que, si l'on applique à son art l'étiquette " art féministe ", c'est seulement pour le pousser hors du champ de l'art conceptuel avec lequel l'intervention féministe a été très liée dans les années 1970. On ne dit pas art « masculiniste » ou art " phallique » ou art « chrétien ». Si l'on me catégorise comme une historienne d'art féministe, il semble que je ne peux parler que de choses féministes: or je parle de l'histoire de l'art, mais en posant des questions nouvelles, qui peuvent enrichir ou fertiliser nos recherches pour ouvrir les questions que l'on a refoulées, réprimées ou évitées. C'est un projet créateur et plus ouvert que de créer une énième catégorie. L'art de Michel-Ange peut lui aussi poser des questions de différence sexuelle. Il a mis sur la voûte de la chapelle Sixtine, au centre d'un monument emblématique du catholicisme, le moment où le dieu homme aime l'homme : c'est donc de l'amour de l'homme que la Création est née. C'est là une interprétation féministe de Michel-Ange, axée non pas sur sa sexualité, mais sur son art. Une création plastique nous enseigne que la Création peut être créée par l'amour d'un homme pour l'homme, pour un autre homme.

J. L. Vous dites que vous n'aimez pas tout le monde, bien évidemment, mais que vous pouvez reconnaître qu'il y a une très grande intelligence artistique de tel artiste, homme ou femme, peu importe, et ne pas l'aimer. Je ne suis pas sûre. Ce n'est pas une critique que je vous adresse, mais je veux dire que si l'on reconnaît qu'il y a une très grande intelligence artistique pour telle ou 
telle raison, etc., est-ce que l'on peut ne pas aimer cet artiste ? C'est aussi une question que je me pose à moi-même.

G. P. Si l'on étudie l'histoire de l'art, on sait que l'on n'arrive pas spontanément à comprendre. On doit étudier plusieurs méthodes et disciplines pour arriver à une connaissance assez profonde pour reconnaître ce que cet artiste ou un autre a fait. Le but de notre discipline est donc d'infléchir cette idée d'un amour de l'art spontané, qui entraîne une reconnaissance immédiate. Pour les femmes, il manque toute la structure, tous les processus pour arriver à une telle reconnaissance, et même les femmes qui les étudient ont été aliénées par leurs propres imaginations. C'est pour ça que j'insiste : avec le féminisme, c'est la première fois dans l'histoire de l'art que je peux arriver à l'universalité pour étudier ma propre voix, même si le territoire du féminin est encore inconnu. Mais je peux devenir, comme Freud, un archéologue qui voyage dans ce paysage inconnu, ce continent noir et sombre pour déchiffrer des signes et des traces. Et c'est toujours un pacte, quelque chose entre ce que je voudrais savoir et ce que j'apporte au processus de production des connaissances ou de la science de mon époque, et ce que je peux rencontrer sans imposer mes projections. Ce processus de se retirer pour découvrir quelque chose d'inouï, d'emporter dans le projet de recherche des choses qui peuvent animer ce qui reste maintenant mort et stupide et sans intérêt ne va pas de soi. Je l'ai fait seulement avec les quelques artistes sur lesquels j'ai travaillé, comme Shoshana Felman l'a fait : ce pacte de lecture est une Nachträglichkeit, un après-coup, du rétrospectif. Mais il n'y avait pas alors, dans nos musées, dans nos bibliothèques, une préservation suffisante des archives et des textes dont on avait besoin pour arriver à une interprétation assez sensible et nuancée, comme on peut l'avoir maintenant pour les artistes masculins valorisés par l'histoire de l'art autorisé. Donc je peux apprendre à aimer, à apprécier des artistes pour lesquelles je n'ai pas eu une attirance instantanée, par l'enseignement, grâce à quoi on a l'occasion de comprendre beaucoup mieux ce qui ne paraît pas très intéressant au premier regard.

PHILIPPE BORDES. Je me demande s'il n'y a pas trois aspects dans votre discours, et je ne vois pas très bien comment ils se relient. À la fin, quand vous parlez d'artistes, j'imagine que vous pensez "artistes contemporains". Parce que je vois trois choses: d'une part, un peu à la manière de Carlo Ginzburg23, vous voulez donner la voix à ceux qui n'ont pas accès à l'expression. Par ailleurs, il me semble que votre discours est fondé sur une conception un peu étroitement muséale de l'art. On a vu dans les années 1970 que tous les penseurs innovants ne travaillaient que sur les artistes sanctionnés par les institutions. Et puis le troisième aspect, c'est ce que vous venez de dire à propos de votre collaboration, on va dire, intellectuelle et empathique avec les artistes. Telle que vous l'avez présentée, j'ai senti que c'était plutôt des artistes contemporains, et l'art contemporain a ses propres protocoles, je dirais, d'explication.

G. P. Je ne pense pas que je donne la voix aux personnes qui n'ont pas de voix. Elles parlent, mais personne n'écoute. C'est différent. C'est parti d'un défi que m'ont lancé mes étudiants qui viennent d'Inde, d'Afrique, d'Amérique latine : pourquoi est-ce que je ne connais pas leurs artistes ? Comment puis-je penser que je suis historienne de l'art si je reste dans une telle ignorance? Donc ce n'est pas que je donne la voix aux minorités ; c'est que je dois écouter ce qu'on peut me dire d'une situation ou d'une autre. Generations and geographies in the visual arts (Londres/New York, 1996), c'était justement le résultat de cette réflexion : changer mon point de vue pour être non, 
comme les Américains, globalisante, mais pour pouvoir m'adresser des questions qui viennent d'une position autre que la mienne.

Ensuite, les effets du féminisme et des autres mouvements sociaux des années 1970 ont vraiment changé la situation : l'art contemporain est différent et plus vaste dans son contenu, et il y a d'autres moyens de l'expliquer; voilà vraiment l'effet de ces défis. On doit élaborer quelque chose de beaucoup plus complexe. J'ai une collègue, Catherine Karkov, qui pratique une intervention féministe sur l'art du Moyen Âge, ce qui est formidable parce qu'il y a beaucoup d'inscriptions de femmes. Si on fait des recherches dans les archives sur les femmes du Moyen Âge ou de la Renaissance, à Bologne ou à Rome, on trouve des traces, on trouve des archives - finalement on n'a jamais été aussi antiféministe que dans la modernité !

Quelque chose est arrivé avec l'art contemporain qui devrait, je pense, intéresser les historiens de l'art : pourquoi est-ce qu'on a tout d'un coup abandonné la peinture? Peut-être que vous vous rappelez l'exposition Face à l'histoire ? ${ }^{24}$ Après les années 1980, la plupart des documents étaient photographiques ou cinématographiques, et l'exposition ne proposait qu'une seule petite section avec un groupe de peintures. Pourquoi la culture a-t-elle abandonné ce moyen de penser le monde, qui a dominé pendant des siècles? Les historiens de l'art ne savent pas comment répondre à ce changement de medium. Et donc, par faiblesse, on retourne à quelque chose de connu et de soi-disant continu, au lieu de chercher les moments vraiment transformateurs.

J. L. Votre critique, c'est que l'historien de l'art ne peut pas tenir compte des transformations que les innovations technologiques induisent dans l'art lui-même. Au fond, est-ce que vous ne demandez pas à l'historien de l'art de se transformer en critique d'art ? Parce que c'est au critique d'art, me semble-t-il, de comprendre ce qui se passe aujourd'hui, de comprendre pourquoi il y a ces nouvelles formes d'art. Est-ce que ce n'est pas attendre beaucoup trop de l'histoire de l'art?

G. P. C'est une question très intéressante. La différence, c'est que les critiques doivent expliquer immédiatement et donc on a un discours de critique. De temps en temps, il devient plus intéressant, plus intellectuel. Tandis que l'étude historique nous donne des perspectives non seulement d'une histoire qui continue, progresse, mais aussi de l'étrangeté des moments de rupture, des manques de continuité, des changements. Pour moi, l'historien de l'art qui veut s'occuper de l'art contemporain ne doit pas être schizophrène - au moment où j'étudie Mary Cassatt, je suis historienne, et au moment où j'étudie Mary Kelly, je suis critique - parce que le critique a toujours une posture historique. On doit toujours avoir ce rôle de légitimation plutôt que de tenir un discours un peu apologétique, tandis que l'historien de l'art met en question, pose peut-être des questions dérangeantes. Ce n'est pas une question d'évaluation et d'interprétation, c'est qu'il s'agit de comprendre le mouvement, le changement des structures. Aujourd'hui la pensée passe par une autre technologie, difficile à identifier clairement. Les différences entre la culture générale et les actes de l'art sont assez difficiles à définir dans ces conditions là. Et c'est pourquoi je pense que nos connaissances historiques, nos possibilités de penser, avec ses grandes perspectives et avec cette profondeur de compréhension de tout ce qui arrive dans l'art, peuvent apporter quelque chose à la connaissance de l'art contemporain qui est vraiment la même chose que la critique. 


\section{NOTES}

1. Voir la bibliographie complète de Griselda Pollock sur www.leeds.ac.uk/cath.

2. Griselda Pollock, Vision and Difference : Feminism, Feminity and the Histories of Art, Londres, (1988) 2003 ; Differencing the Canon: Feminist Desire and the Writing of Art's Histories, Londres, 1999.

3. Mary Kelly, «Art and Sexual Politics » (1976), réédité dans Rozsika Parker, Griselda Pollock, Framing Feminism: Art and the Women's Movement 1970-1985, Londres, (1987) 1992.

4. Griselda Pollock, «Can Feminism Survive the Impact of Feminism? », conférence à la CAA, New York, 1989, reprise dans la préface de Vision and Difference, Londres, 2003.

5. Frederic Jameson, The Political Unconscious : Narrative as a Socially Symbolic Act, Ithaca (NY), 1982.

6. Teresa de Lauretis, Technologies of Gender: Essays on Theory, Film and Fiction, Londres, 1987.

7. Franz Kugler, Handbuch der Geschichte der Malerei, 2 vol., Stuttgart, 1837; Franz Kugler, Handbuch der Kunstgeschichte, 2 vol., Stuttgart, 1841-1842; Charles Blanc, Histoire des peintres de toutes les écoles, Paris, 1858.

8. L'article de Joan W. Scott, "Genre : une catégorie utile d'analyse historique ", dans Jeanne Bisilliat, Christine Verschuur éd., Le genre: un outil nécessaire, introduction à une problématique, Paris, 2000 ["Gender: A Useful Category of Historical Analysis », American Historical Review 91/5, décembre 1986 (traductions en français, italien, espagnol et portugais)] est un texte fondateur. Voir aussi Donna J. Haraway, « 'Gender' for a Marxist Dictionary: The Sexual Politics of a Word », dans Donna J. Haraway, Simians, Cyborgs and Women; The Reinvention of Nature, Londres, 1992, p. 127-148 et Gayle Rubin, « The Traffic in Women: Notes on the 'Political Economy' of Sex », dans Rayna Reiter éd., Toward an Anthropology of Women, New York, 1975.

9. Simone de Beauvoir, Le deuxième sexe, Paris, 1949.

10. Scott, 1986, cité n. 8 .

11. Pierre Bourdieu, De la domination masculine, Paris, 1998.

12. Judith Butler, Gender Trouble : Feminism and the Subversion of Identity, New York, 1990 ; trad. fr. : Trouble dans le genre, Paris, 2005.

13. Adrienne Rich, «Towards a Woman Centred University " (1973-1974), dans Lies, Secrets and Silence: Selected Prose 1966-1978, Londres, 1979, p. 125-156.

14. Si le genre n'existe pas comme fait accompli, on doit nous permettre d'imaginer ces inscriptions dans la culture comme produit des subjectivités qui ne sont pas fixées. Julia Kristeva a suggéré que le féminin doit être compris comme position de négativité créatrice qu'on découvre par exemple dans les textes de Mallarmé et d'autres poètes de l'avant-garde. Monique Wittig propose l'idée qu'il existe des écrivains féminins qui ne sont pas des femmes: les lesbiennes, selon elle, résistent à la position de classe nommée «femme » dans l'économie patriarcale. On peut donc imaginer la position d'un même refus des normes masculines avec ce néologisme «non-homme ». On voudrait échapper à la substitution d'une identité fixe pour une autre. La fluctuation des identités qui est le résultat de nos formations psychiques et linguistiques peut être mobilisée par les pratiques sémiotiques et esthétiques.

15. Hélène Cixous, «Le rire de la Méduse ", dans L'arc, $n^{\circ} 61,1975$.

16. Griselda Pollock, éd., Generations and Geographies in the Visual Arts: Feminist Readings, Londres/ New York, 1996.

17. L'idée de singularité, qu'on attribue au philosophe médiéval Duns Scot, figure dans plusieurs ouvrages récents de Julia Kristeva, par exemple, Le génie féminin: Arendt, Klein, Colette, Paris, 1999-2001, ou "Le temps des femmes", dans Cahiers de recherche de sciences des textes et des documents, 5, 1979, p. 5-19.

18. "I will suggest that none of us, as women, has as yet, precisely, an autobiography. Trained to see ourselves as objects, to be positioned as other, estranged to ourselves, we have a story that 
cannot, by definition, be self-present to us: a story that, in other words, is not a story, but must become a story. And it cannot become a story except through a bond of reading, that is, through the story of the other, the story read by other women, the story of other women, that story told by others, insofar as this story of the other, as our own autobiography, has as yet precisely to be owned. I will suggest that it cannot be owned by our attempting any direct access to ourselves as women - getting personal - or by pretending to leave culture to step outside the text. Rather, I will here propose that we might be able to engender, to access our story only indirectly, by conjugating literature, theory and our autobiography together through the act of reading and by reading, thus, into the text of culture, at once our sexual difference and our autobiography as missing ", Shoshana Felman, What Does a Woman Want? Reading and Sexual Difference, Baltimore, 1993, p. 14.

19. Kristeva, 1979, cité n. 17, p. 5-19.

20. Hannah Arendt, The Human Condition, Chicago, 1958 ; trad. fr. : La condition de l'homme moderne, Paris, 1961.

21. Henrik Holm, «Contest-nation: Denmark - A PTSD-struck Contesting Analysis », (colloque, Belfast, avril 2007), à paraître dans Griselda Pollock éd., The Visual Politics of Psychoanalysis in a Post-traumatic World.

22. Heather Dawkins, «Frogs, Monkeys and Women: A History of Identifications across a Phantastic Body ", dans Richard Kendall, Griselda Pollock éd., Dealing with Degas: Representation of Women and the Politics of Vision, Londres, 1991, p. 202-217.

23. Carlo Ginzburg, Il fromaggio e i vermi: il cosmo di un mugnaio del'500, Turin, 1976 ; trad. fr. : Le fromage et les vers : l'univers d'un meunier du Xvi siècle, Paris, 1980.

24. Face à l'Histoire, 1933-1996. L'artiste moderne devant l'événement historique, Jean-Paul Ameline, Harry Bellet éd., (cat. expo., Paris, Centre Georges-Pompidou, 1996), Paris, 1996.

\section{INDEX}

Keywords : art history, historiography, feminist art history, feminism, male canon, phallocracy, gender studies, queer studies

Mots-clés : histoire de l'art, historiographie, histoire de l'art féministe, féminisme, canon masculin, phallocratie, gender studies, queer studies

\section{AUTEURS}

\section{GRISELDA POLLOCK}

Née en Afrique du Sud et ayant vécu au Canada anglophone et au Québec, elle a poursuivi sa formation en Grande-Bretagne (Oxford University et Courtauld Institute of Art). Nommée enseignante à l'université de Leeds en 1977, elle y dirige depuis 2001 le Centre for Cultural Analysis, Theory and History. À partir des questions de genre, de race et de classe et de leur impact sur le modernisme et les pratiques artistiques depuis la fin du XIX ${ }^{\mathrm{e}}$ siècle en Europe et aux États-Unis, elle a contribué à mettre en valeur les apports de la théorie psychanalytique et du féminisme dans une approche ouverte de l'histoire de l'art, et élargi ses réflexions sur les 
problématiques des arts visuels dans les cultural et post-colonial studies. Elle vient de publier Museums after modernism strategies of engagement (Malden, 2007). 